\title{
Alternativa para la generación de gas natural sintético a partir de una fuente de energía renovable mediante tecnología "Power to Gas" en Colombia
}

\author{
Edgar A. Vidal G*; Carlos A. Fontalvo G. \\ Escuela de Ingeniería de Petróleos, Universidad Industrial de Santander (UIS), Carrera 27 Calle 9. Bucaramanga, Colombia. \\ *E-mail: edgarvida194@gmail.com
}

\begin{abstract}
Resumen
La actualidad de la industria de los hidrocarburos gaseosos en Colombia en torno a, la disminución de reservas y el consumo creciente de gas natural en el país, la disminución en la relación reservas/consumo, conlleva a una futura incertidumbre sobre las perspectivas energéticas en el mercado del gas natural. Es por esto, que el país está incursionado en sectores alternos, los cuales buscan brindar una solución a esta problemática, permitiendo así, la innovación en otras áreas.
\end{abstract}

La tecnología Power to Gas (PtG) transforma la energía eléctrica provenientes de fuentes renovables en energía química a través de la electrólisis. El producto obtenido de la electrólisis es el hidrógeno, que posteriormente es utilizado en la metanación para la producción de metano sintético.

El gas sintético obtenido a través del proceso Power to Gas, tiene la ventaja de poder ser usado con funciones semejantes a la del gas natural existente y así mismo puede ser comprimido, almacenado o inyectado en redes de transporte y distribución de gas natural. PtG es una alternativa para la generación de gas, basado en el mediano a alto desarrollo en sistemas denominados "limpios".

Palabras clave: Gas, Gas Natural Sintético, Energía Renovable, Electrólisis, Hidrógeno, Metanación, Power to Gas, Almacenamiento de gas.

\section{Alternative solution to synthetic natural gas generation from a source of renewable energy through "Power to Gas" technology}

\begin{abstract}
The present of the gaseous hydrocarbon industry in Colombia is facing a new challenge, the limited availability of reserves and the potential growth in consumption of gas. This is why the country is venturing in alternative sectors that can provide a solution to this situation and allows the innovation in other areas.

Power to Gas (PtG) transforms electrical energy into chemical energy through electrolysis. The product obtained from electrolysis is hydrogen and is continuously used in methanation for the production of synthetic methane.

The synthetic gas obtained through Power to Gas has similar properties to natural gas and can also be compressed, stored or injected into the same transport and distribution networks. PtG is an alternative to gas generation, through the storage of energy from green technologies.
\end{abstract}

Keywords: Gas, Synthetic Natural Gas, Renewable Energy, Electrolysis, Hydrogen, Methanation, Power to Gas, Gas Storage.

Cita: Vidal, E. A. y Fontalvo, C. A. (2018). Alternativa para la generación de gas natural sintético a partir de una fuente de energía renovable mediante tecnología "Power to Gas" en Colombia. Revista Fuentes: El reventón energético, 16(1), 71-79. 


\section{Introducción}

El concepto de Power to Gas involucra a uno de los mayores problemas de nuestros sistemas energéticos: el del almacenamiento de la electricidad. El crecimiento de la demanda eléctrica en el país muestra que es de suma importancia el sector energético con relación a la generación de hidrocarburos para la economía nacional, en promedio la demanda eléctrica tuvo una aceleración anual del 2,9\% en los últimos 4 años; en sintonía con el crecimiento de la economía colombiana que fue de 4\%. (UPME, 2016. p. 17)

El almacenamiento de electricidad es posible cuando la producción excede la demanda durante algunos periodos de tiempo. Los procesos de almacenamiento convencionales debido a las limitaciones técnicas, los costos implicados, su baja eficiencia, y tiempo de vida, los hacen inadecuados para el almacenamiento de grandes cantidades de energía por un tiempo prolongado. (CHBC, 2015. p. 6)

La alternativa de generar gas natural sintético a partir de una fuente de energía renovable, surge debido a la falta de reservas y proyecciones estimadas (UPME, 2016), que sugieren que la demanda de gas natural para el 2023 va a superar la oferta, en consecuencia a la falta de recursos renovables y nuevos grandes descubrimientos, que permitan prolongar la llegada de un superávit de gas (Giraldo, Calle \& Pineda, 2009).

\section{Balance de gas natural en Colombia}

PROMIGAS (2016) estima que. "A nivel nacional la demanda de gas natural en el escenario medio alcanzará un crecimiento promedio año de 2,2\% entre 2015 y 2035, pasando de 1.060 GBTUD a 1.707 GBTUD (12,944 GWe a 20,84 GWe), impulsada por el crecimiento económico, aumento de población y sustitución de algunos energéticos menos eficientes por gas natural, en cumplimiento de las recomendaciones ambientales de la reunión de Paris del año 2015" (p. 37).

Haciendo un análisis a las proyecciones de la UPME sobre la demanda de gas natural, se prevé que en el año 2023 habrá un equilibrio entre la oferta y la demanda. Suponiendo diferentes escenarios de alta, media y baja oferta, en comparación con diferentes tendencias de demanda, con lo cual se obtiene una fecha límite, debido a la curva media de proyección, basados en el consumo de gas natural. El estudio fue realizado, teniendo en cuenta las diferentes áreas de consumo de gas como el residencial, comercial, industrial, petroquímico, petróleo, transporte y termoeléctrico, así como la producción de gas en los diferentes campos del país, además, teniendo en cuenta el desarrollo de reservas probables y reservas posibles (Vaca, 2001) y la incorporación de nuevos recursos, tanto convencionales como no convencionales en el país (Gómez, 2001).

La incorporación de la tecnología Power to Gas, podría suponer una extensión en el periodo en el cual se llegará al superávit o déficit de gas natural, ampliando la curva de proyección, mediante la generación de gas sintético, comercializado a través de las redes de gas, la cual cuenta con una capacidad máxima de mediano plazo de 2,5 GPCD o 70,79 $\mathrm{Mm}^{3} / \mathrm{d}$ (PROMIGAS, 2017. p. 49)

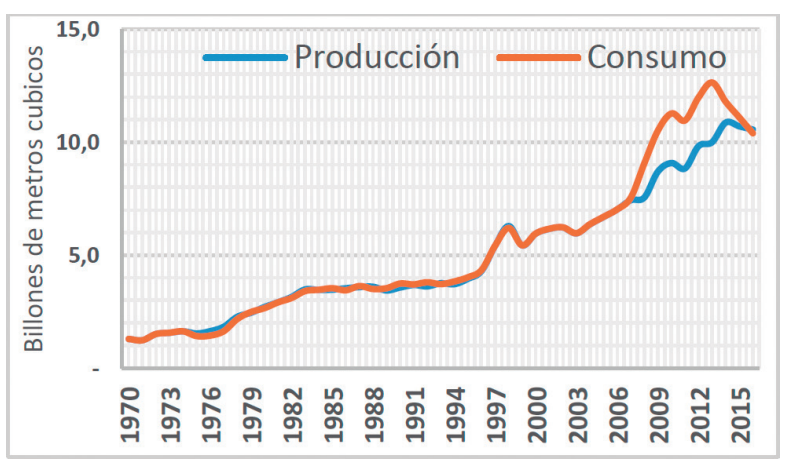

Figura 1. Producción vs Consumo de gas natural en Colombia. Fuente: (UPME, Balance Gas Natural, 2016)

\section{Power to Gas}

Las energías renovables y la temática que involucra la reducción de contaminantes del medio ambiente son temas tratados hace varias décadas por el gremio científico alrededor del mundo (Acevedo, Jaramillo \& Abril, 2013). El proceso de electrólisis, que es uno de los métodos más antiguos utilizados para la disociación o separación de un compuesto por medio de la electricidad, y la metanación, que fue descubierta por Paul Sabatier y Jean Senderens, la cual consiste en la síntesis química de metano a partir de la interacción de hidrogeno, monóxido de carbono y dióxido de carbono. Estas dos técnicas se integran para formar la tecnología Power to Gas (PtG) que es estudiada y desarrollada en primicia por el Dr. Sci. Koji Hashimoto de la universidad de Tohoku. (Hashimoto, et al, 2016).

Los rangos de eficiencias esperados para el aprovechamiento de la energía eléctrica proveniente de fuentes renovables son representados en la tabla 1. En esta, se presentan tres condiciones en la conversión de electricidad a hidrógeno y posteriormente a gas natural sintético. En esta etapa en promedio se presenta un mínimo de eficiencia de $49 \%$ y un máximo de $77 \%$. 
Tabla 1. Eficiencia conversión energía eléctrica a gas. Fuente: (Sterner, 2009. p.210)

\begin{tabular}{|c|c|c|}
\hline Etapas & Eficiencia(\%) & Condiciones limites \\
\hline \multicolumn{3}{|l|}{ Electricidad a gas } \\
\hline $\begin{array}{l}\text { Electricidad } \rightarrow \text { Hidrógeno } \\
\text { Electricidad } \rightarrow \text { Metano }(\mathrm{SNG})\end{array}$ & $\begin{array}{l}54-72 \\
49-64\end{array}$ & $\begin{array}{c}\text { Almacenamiento } \\
\text { - Incluyendo } \\
\text { compresión a } 200 \text { bar }\end{array}$ \\
\hline $\begin{array}{l}\text { Electricidad } \rightarrow \text { Hidrógeno } \\
\text { Electricidad } \rightarrow \text { Metano }(\mathrm{SNG})\end{array}$ & $\begin{array}{l}57-73 \\
50-64\end{array}$ & $\begin{array}{l}\text { Transporte - } \\
\text { Incluyendo } \\
\text { cmpresión a } 80 \text { bar }\end{array}$ \\
\hline $\begin{array}{l}\text { Electricidad } \rightarrow \text { Hidrógeno } \\
\text { Electricidad } \rightarrow \text { Metano }(\mathrm{SNG})\end{array}$ & $\begin{array}{l}64-77 \\
51-65\end{array}$ & Sin compresión \\
\hline
\end{tabular}

\section{Electrólisis}

Electrólisis es el proceso mediante el cual se utiliza la energía eléctrica para producir cambios químicos, mediante una reacción no espontánea de óxidoreducción. La corriente eléctrica pasa a través de un electrolizador o celda electrolítica, donde se lleva a cabo el proceso de separación, siendo este uno de los principales métodos químicos de separación. Una de las principales ventajas de la utilización de la electrólisis como método de separación es que no es necesario aumentar la temperatura para que la reacción tenga lugar, evitando asi pérdidas energéticas y reacciones secundarias.

Mediante de este proceso se logra obtener diferentes elementos a partir de compuestos, como por ejemplo (cloro, hidrógeno, oxígeno), la purificación de metales (el mineral metálico se disuelve en ácido, obteniéndose por electrólisis el metal puro), o la realización de recubrimientos metálicos, protectores y con fines decorativos.

En teoría para generar $1 \mathrm{~kg}$ de hidrógeno se requiere de $40 \mathrm{kWh}$ de electricidad. Además, teóricamente la descomposición del agua requiere de un voltaje de 1,23V (que corresponde a la descomposición energética teórica de $286 \mathrm{~kJ} / \mathrm{mol}$ o $15,9 \mathrm{MJ} / \mathrm{kg}$ a $25{ }^{\circ} \mathrm{C}$ ) generalmente las celdas cuentan con un voltaje de 2V. Gotz (2015) afirma. "Según su fabricación los electrolizadores pueden operar entre el $20 \%$ y el $100 \%$ de su capacidad de diseño y hasta una sobrecarga de operación de $150 \%$ lo cual hace a la tecnología una excelente opción para el proceso Power to Gas que depende de las fluctuaciones y del intermitente suministro de energía” (p. 1371).

Existen tres tipos diferentes de tecnologías de interés para el proceso de electrólisis en Power to Gas:
- Celdas para electrólisis alcalina del agua (AEL)

Es considerada una tecnología madura, con varias décadas de productos comercializados para la producción de hidrógeno. La electrólisis alcalina, puede ser descrita como el uso de una corriente eléctrica que pasa a través de una celda electrolizadora, causando la descomposición del agua, para generar hidrogeno en el cátodo de la celda. En la celda, una solución de $(\mathrm{KOH}$ o $\mathrm{NaOH})$, es usada como electrolito, en el cual dos electrodos están completamente inmersos en él, con un diafragma micro poroso como elemento separador entre las regiones catódicas y anódicas. La electrólisis alcalina funciona a presión atmosférica y a presiones elevadas.

- Celdas para electrólisis por membrana de electrolito polimérico (PEM)

Comparada con la electrólisis alcalina, la electrólisis por membrana es una tecnología relativamente nueva, ha estado en continuo desarrollo desde 1950 y en 1978 fue puesto en venta el primer electrolizador de este tipo. La tecnología es basada en una membrana polimérica sólida, siendo esta, la principal diferencia con el electrolizador alcalino, son membranas conductoras muy finas, con espesores de entre 50 y $250 \mu \mathrm{m}$. El agua fluye por un lado del ánodo y es oxidada electroquímicamente para formar oxígeno, iones de hidrogeno y electrones. Los iones de hidrogeno migran a través de la membrana y se recombinan con los electrones, debido al circuito externo, para formar gas hidrógeno en el cátodo.

- Celdas para electrólisis a base de óxidos solidos (SOEC)

La electrólisis de óxidos solidos también conocida como electrólisis de alta temperatura, es la más reciente de las tecnologías desarrolladas para los procesos de electrólisis y continua en etapa de laboratorio. En la electrólisis de óxidos solidos se utiliza óxido de circonio $\left(\mathrm{ZrO}_{2}\right)$ mezclado con $8 \mathrm{~mol} \%$ de óxido de itrio $\left(\mathrm{Y}_{2} \mathrm{O}_{3}\right)$ como electrolito, que a elevadas temperaturas es altamente conductor de iones de oxígeno, el cual posee una buena estabilidad térmica y química (Gotz, Bajohr, Kolb, Lefebvre y Reimert, 2015).

Las altas temperaturas se utilizan para reducir el voltaje de equilibrio de la celda, y así, la demanda de electricidad. Una de las ventajas más significativas del electrolizador por óxidos sólidos es la baja demanda energética que este requiere. 


\section{Metanación}

Los conceptos de metanación con $\mathrm{CO}$ y $\mathrm{CO}_{2}$ fueron descubiertos por los químicos Paul Sabatier y Jean Senderens en 1902 y han sido investigadas por más de 100 años. La aplicación más importante fue la eliminación del monóxido de carbono de gases sintéticos, de este modo gano importancia en la producción de gas natural sintético cuando se presentó la crisis del petróleo en la década de 1970 (Rönsch, et al, 2015. p.279). Luego de varios años de grandes desarrollos para la metanación, al igual que periodos de tiempo de estancamiento; en el siglo XXI con el acelerado crecimiento del cuidado ambiental y gracias a la necesidad de reducir los gases contaminantes generadores del efecto invernadero fue reanimada la investigación de los procesos de metanación.

Las reacciones de metanación son inherentes a la síntesis química o biológica del metano a partir de la interacción entre el hidrógeno $\left(\mathrm{H}_{2}\right)$, monóxido de carbono $(\mathrm{CO})$ y dióxido de carbono $\left(\mathrm{CO}_{2}\right)$. La metanación es uno de los procesos químicos importantes y necesarios para la obtención de metano sintético en la tecnología Power to Gas.

La denotación "sintético" en la tecnología PtG hace referencia a la producción de un gas natural rico en metano $\left(\mathrm{CH}_{4}\right)$ pero que tiene un porcentaje de otros componentes como $\mathrm{H}_{2}, \mathrm{CO}, \mathrm{CO}_{2}, \mathrm{H}_{2} \mathrm{O}$ y que fue obtenido a través de un proceso de refinado o una tecnología no convencional como lo es la tecnología Power to Gas.

Como afirma Schaaf (2014), "en términos químicos asumiendo que aproximadamente en el proceso a una velocidad del gas por hora (GHSV) de 5.000 h-1 y la conversión total del $\mathrm{CO} 2$, es necesario eliminar $2 \mathrm{MW}$ de calor por litro de catalizador. Como consecuencia, en la metanación es de suma importancia controlar la temperatura que en rango está entre $200^{\circ} \mathrm{C}$ a $550^{\circ} \mathrm{C}$, con el fin de, prevenir limitaciones termodinámicas y síntesis del catalizador en el reactor" (p. 14).

Las presiones de operación de los reactores para la reacción de catalización en la metanación son de (1 a $100 \mathrm{~atm})$. La temperatura y la presión son factores que afectan la eficiencia del proceso, generando que a mayor temperatura la fracción molar de metano en el gas producido sea menor y para la presión, a mayor presión se aumenta la fracción molar de metano en el gas producido.

Actualmente, existen tres tipos de tecnologías para la generación de gas mediante el proceso de metanación en un sistema Power to Gas:
- Metanación de lecho fijo

Este método utiliza el catalizador en forma de gránulos, que varían en diámetros de $0,3-0,7 \mathrm{~cm}$ y se encuentran dentro del reactor generando un lecho catalizador homogéneo y fijo (estático). Un gran número de productos de gases se reciclan a través del reactor para reducir la concentración del gas reactante, este disminuye la velocidad de metanación y aumenta la eficiencia de eliminación de calor. La principal limitante de la tecnología es precisamente la transferencia de masa entre los gases y el catalizador sólido ubicado dentro del reactor. El tiempo de vida promedio de los catalizadores empleados es de 4 años.

En la reacción catalítica, reactor de lecho fijo tiene la ventaja sin precedentes debido al tiempo de residencia controlable, es de fácil operación, así como la viabilidad para acortar el ciclo de desarrollo. Este sistema de lecho fijo trabaja con altas condiciones de temperatura al abarcar reacciones exotérmicas por lo que el control de la temperatura es una variable indispensable para el control. Los focos y cambios de temperatura pueden degradar o dañar el catalizador utilizado en el proceso. Sin embargo, debido a la fuerte naturaleza exotérmica de la reacción de metanación y la transferencia de calor radial y axial limitada en un reactor de lecho fijo, es difícil de operar un solo reactor de lecho fijo en condiciones isotérmicas.

Para un buen control de la temperatura de reacción, por lo menos dos reactores adiabáticos tienen que ser conectados en serie. El control de temperatura se puede realizar mediante la recirculación de las corrientes de gas de salida del reactor y por etapas de enfriamiento de gases intermedios.

\section{- Metanación con lecho fluidizado}

En este método, la reacción de metanación se lleva a cabo dentro de un lecho fluidizado con partículas del catalizador. Se genera una mezcla óptima entre las partículas de gas y del catalizador sólido en el lecho fluidizado, lo que ocasiona que se puedan conseguir condiciones relativamente isotérmicas; además de alta transferencia de masa y calor.

El muy buen control del proceso que se presenta en este reactor lo caracteriza como un sistema eficiente para el desarrollo de las reacciones que involucra. Por lo tanto, la optimización del proceso en este reactor debido a las condiciones termodinámicas permite que se empleen configuraciones más simplificadas en comparación con 
los de lecho fijo, ya que no se requieren sistemas de reactores en serie.

La colisión entre partículas en este sistema desempeña un papel importante en el intercambio de calor, la turbulencia generada con el lecho del catalizador aumenta las condiciones del contacto entre las partículas y los gradientes de temperatura son minimizados. Sin embargo, la abrasión y el arrastre de partículas de catalizador en la corriente de gas es el mayor problema que presenta porque genera daños en las partes internas del reactor.

\section{- Metanación de tres fases}

Esté método desarrolla un proceso de transferencia de calor a través de un reactor lleno de un líquido que permite un control eficaz y preciso de la temperatura. Debido a la capacidad de calor adicional de su fase líquida, un reactor de tres fases es menos propenso a cambios de temperatura rápidos en el caso de la fluctuación de corrientes de alimentación. Además, la metanación en tres fases permite la eliminación del catalizador durante la operación; esto conduce a una operación isotérmica estable del proceso de metanación.

Las posibles desventajas de un reactor de tres fases incluyen la evaporación o descomposición del líquido de transferencia de calor, así como la resistencia de transferencia de masa adicional debido a la fase líquida.

El proceso de metanación es desarrollado involucrando un sistema de tres fases donde se presentan: un catalizador en estado sólido, reactantes en estado gaseoso y un líquido para la transferencia de calor. El concepto fue diseñado por Chem Systems Inc. En la década de 1970, el reactor operó aproximadamente a 1000 psig $(6894,76$ Kpa) y donde el catalizador fue suspendido en una fase líquida inerte permitiendo la transferencia de calor y el control de la temperatura.

\section{Generación de energía eléctrica mediante fuentes renovables en Colombia}

Las fuentes de energía renovable son un desafío para el desarrollo tecnológico e ingenieril de los científicos alrededor del mundo, como plan de contingencia a los problemas generados por el cambio climático producto de la contaminación y deterioro del medio ambiente. En su mayoría, los países con altos estándares en inversión centran su atención en el crecimiento de sistemas modernos e innovadores que permitan aumentar sus conocimientos y desarrollar tecnologías para la producción de energía eléctrica mediante fuentes renovables para el bienestar del ser humano.

En Colombia, el desarrollo de tecnologías que involucran fuentes de energía renovable ha sido lento, a pesar de ser un país que posee matriz energética tanto en combustibles fósiles como en recursos renovables.

Colombia basa su integración energética en tres fuentes esencialmente, los combustibles fósiles, las termoeléctricas; que requieren del anterior para su generación de energía y, por último, la principal fuente de energía renovable desarrollada; la energía hidráulica con una participación en la capacidad instalada para la generación de energía eléctrica en el país del $70 \%$. Por esta razón, otras fuentes de energía como la eólica, solar y geotérmica no tienen una amplia trayectoria y su implementación no está en la actualidad consolidada.

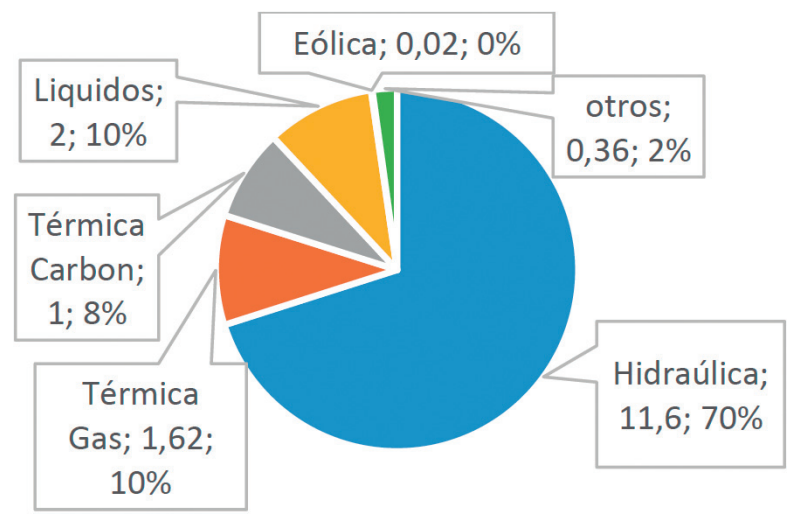

Figura 2. Producción de energía eléctrica en Colombia (GW)

Colombia es un país que depende exclusivamente de dos procesos para la generación de energía eléctrica, estos son, las centrales hidráulicas y las termoeléctricas; que requieren de combustibles para la producción de energía. Las centrales hidroeléctricas representan el $53,58 \%$ de la energía eléctrica generada, en comparación con las termoeléctricas que suministran el $45,42 \%$ de la demanda energética del país. Según esto, Colombia es un país que aún depende de los combustibles para cumplir con el abastecimiento energético, por lo tanto, las fluctuaciones en los recursos naturales ocasionan una intermitencia eléctrica en donde otro proceso debe ajustar la generación de energía.

La implementación de un proceso Power to Gas, supone el desarrollo conjunto y la apuesta por la obtención de energía renovable a partir de diferentes fuentes. Colombia es un país con alta biodiversidad y posee diferentes tipos de ecosistemas interesantes que servirían como escenario para el desarrollo y la producción de energía limpia. 


\section{Sinergia del proceso}

La cadena de producción de gas natural, inicia con la utilización de excedentes de energía eléctrica para su almacenamiento en forma de gas natural sintético. La energía eléctrica se introduce al proceso y mediante la electrolisis, se encarga de disociar las moléculas de agua, en hidrogeno $\left(\mathrm{H}_{2}\right)$ y oxigeno $\left(\mathrm{O}_{2}\right)$.

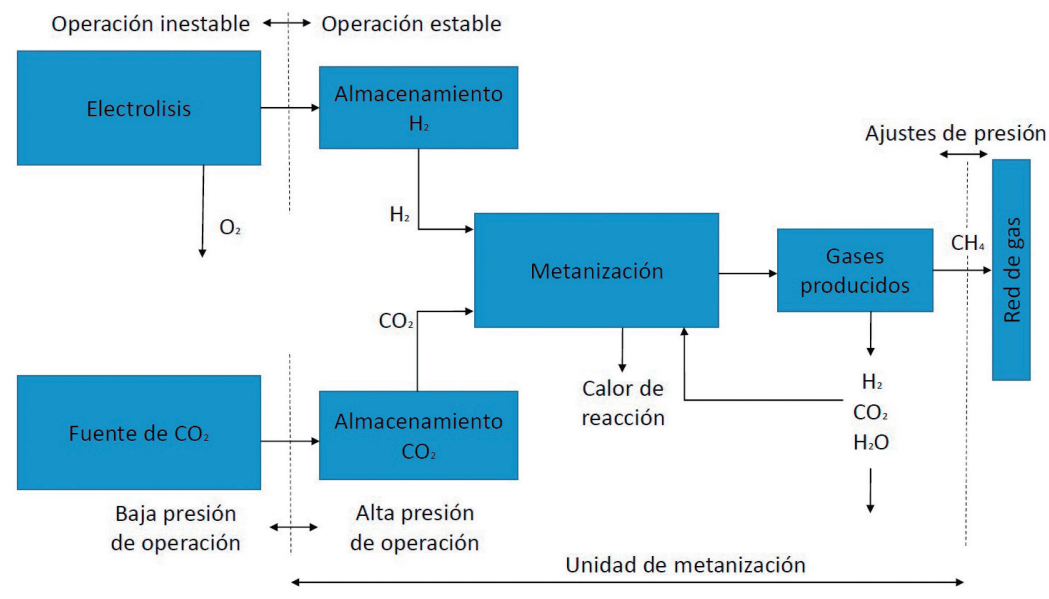

Figura 3. Esquema del proceso $\mathrm{PtG}$

El hidrógeno aunque no es un combustible fósil, últimamente ha ganado mucha popularidad y se han adelantado diferentes procesos e investigaciones para la obtención de hidrogeno de manera más económica. $\mathrm{Su}$ poder energético y las ilimitadas fuentes de hidrógeno en el mundo lo hacen ser un elemento atractivo para ser utilizado como combustible automotriz. El costo asociado a su producción y transporte no ha permitido el desarrollo masivo de centrales productoras. Resulta interesante contar como subproducto del proceso con una sustancia de estas características.

La metanación es un proceso que se realiza posterior a la obtención del hidrogeno, en un metanizador reaccionan el dióxido de carbono y el hidrogeno, produciendo metano sintético en una reacción exotérmica. En teoría, obtendríamos un metano con una pureza del 99\%. La producción de metano sintético serviría como alternativa de mitigación a una futura escases de gas natural.

Para la realización de un proyecto la zona seleccionada debe contar con diferentes atributos técnicos para la realización de cada una de las operaciones, contar con un abastecimiento continuo de cada uno de los suministros requeridos para el proceso, mantener los costos de producción del gas natural sintético en valores que sean competitivos a nivel comercial, entre otros. La figura 4, muestra un planteamiento ilustrativo de las variables esenciales a tener en cuenta para el desarrollo de un proceso PtG.
La generación de energía eléctrica en Colombia depende en su gran mayoría $(70 \%)$ de las centrales hidroeléctricas, las cuales se posicionarían como foco para el desarrollo de cualquier proyecto de gran envergadura. (Hernández, 2011. p.30).

Colombia cuenta con termoeléctricas con capacidades que están entre los 900-441 MW. Estos valores se acercan a la capacidad de generación de las principales hidroeléctricas en Colombia. Su uso para el almacenamiento de energía en forma de gas natural, sería un proceso de reconversión, ya que la obtención de energía por medio de este tipo de centrales consiste en la quema de hidrocarburos, gas natural o carbón.

Por otro lado, hay diferentes proyectos para la construcción de diferentes parques tecnológicos para el aprovechamiento de los recursos naturales en el territorio nacional. La alta dependencia del país entorno a la hidroenergia lo hacen susceptible a diferentes crisis energéticas, debido a la vulnerabilidad de que presenta este tipo de energía por el cambio climático y las sequias que se presentar en diferentes etapas del año.

El fenómeno del niño en 1992 y 1993 o los altos precios experimentados entre 2009 y 2014 son un ejemplo de los problemas asociados a la dependencia de una fuente de energía. El parque eólico jipirachi, un piloto puesto en marcha en el 2004, es un ejemplo de desarrollo e investigación, lo cuales han reafirmado el potencial en fuentes no convencionales de energía renovables en Colombia. 


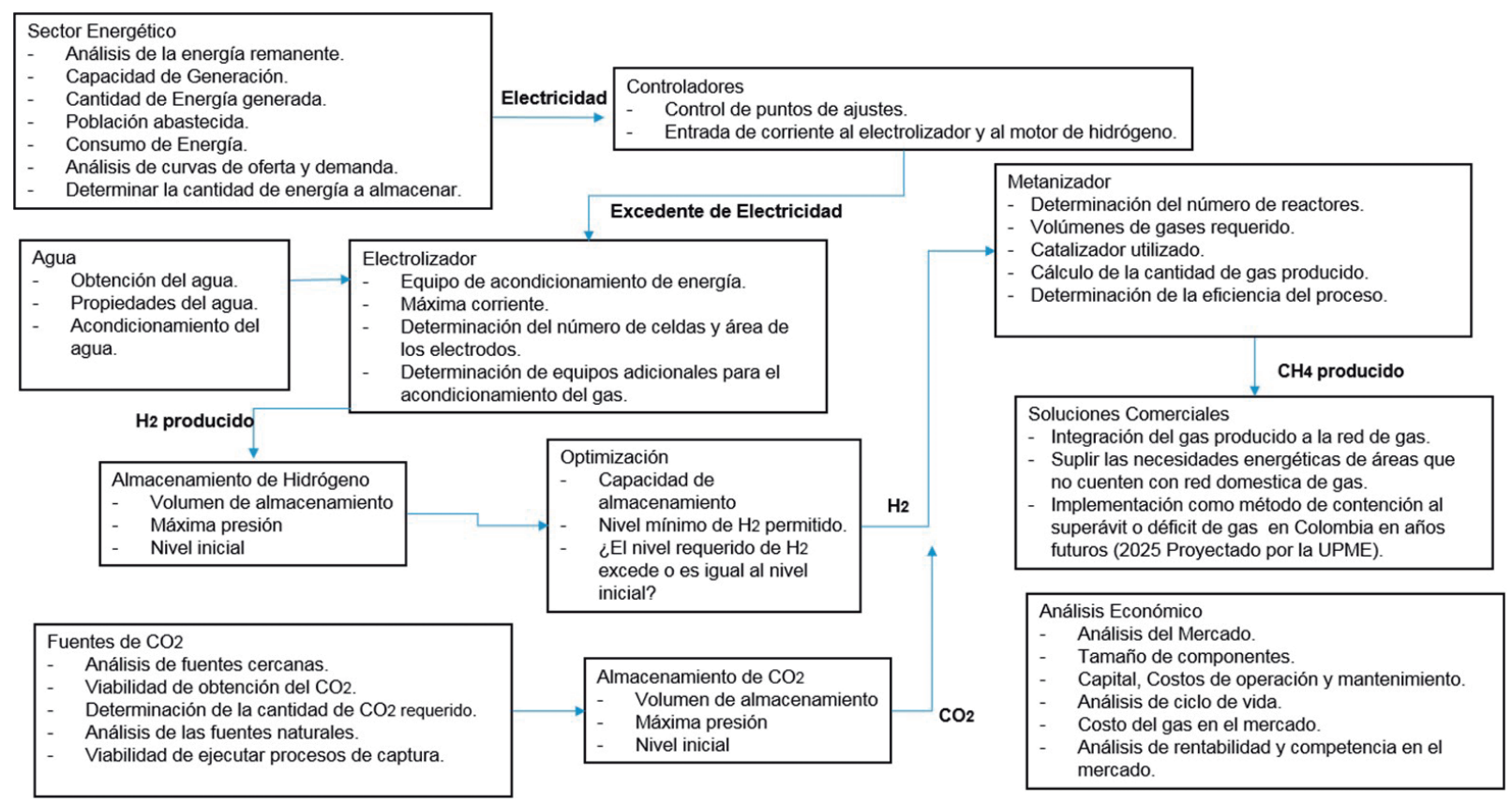

Figura 4. Mapa de ruta para el planteamiento del diseño de una planta PtG.

La alta guajira por su potencial eólico el cual concentra los mayores regímenes de vientos alisios que recibe el país con velocidades promedio cercanas a los $9 \mathrm{~m} / \mathrm{s}$, los cuales se estiman representa un potencial energético del orden de $18 \mathrm{GW}$. Antioquia el departamento con mayor capacidad instalada $(4.5 \mathrm{GW})$ para la producción de energía mediante centrales hidroeléctricas, que también cuenta con el proyecto más ambicioso de Suramérica a nivel hidroeléctrico (hidroituango), junto a Cundinamarca, Boyacá y Santander por su capacidad instalada y desarrollo energético podrían ser foco de estudio para el desarrollo de la tecnología Power to Gas.

Colombia cuenta con un sistema interconectado para el transporte de gas natural que comunica gran parte del territorio nacional, el proceso de distribución y comercialización del gas sintético, no sería diferente al estipulado para el gas natural. Las bajas trazas de impurezas del gas natural sintético hacen que sea un gas que en esencia no requiere de tratamiento para su transporte y comercialización mediante gasoducto. Las zonas con mayor capacidad de generación de energía eléctrica del país se encuentran vinculadas tanto en el sistema eléctrico nacional, como a la red de gasoductos los cuales comunican gran parte del país para la distribución del gas sintético.

El agua es la sustancia líquida por excelencia para el ser humano. En el planeta tierra se estima un volumen aproximado de 1.386 millones de kilómetros cúbicos de agua incluyendo todas las fuentes, entre estas, océanos, mares, ríos, lagos, glaciares, etc. En la actualidad, existen diferentes sistemas para la obtención de agua como captura de represas o embalses por motores, distribución por redes o tuberías, y transporte por carros cisterna.

Para el proceso de electrólisis, se dispone de agua para la obtención del hidrógeno a través de una celda electrolítica. Para esto es necesario el uso de agua con composiciones y parámetros regulados (agua destilada tipo II), para no alterar la composición de los gases obtenidos en el proceso $\left(\mathrm{H}_{2} \mathrm{y} \mathrm{O}_{2}\right)$. Se contempla que las fuentes de agua utilizadas deben ser de alta pureza, comprar un suministro continuo, utilizar el agua de las represas, tratar y aprovechar el agua de producción de campos como Rubiales, diferentes opciones cuya viabilidad depende de la ubicación del proyecto y de los costos asociados a la obtención y tratamiento del agua.

En el mundo existen dos tipos diferentes de fuentes de $\mathrm{CO}_{2}$, las naturales y las antropogénicas. Las fuentes naturales representan el mayor porcentaje de $\mathrm{CO}_{2}$ liberado hacia a la atmosfera. Dentro de las fuentes naturales de $\mathrm{CO}_{2}$ se incluyen la descomposición de la materia, incendios forestales, emisiones desde el océano, emisiones producto de erupciones volcánicas, la respiración de plantas y animales. A través de las actividades humanas y a partir del inicio de la revolución industrial las emisiones de $\mathrm{CO}_{2}$ han aumentado considerablemente. (IPCC, 2005. p.21). 
Las fuentes antropogénicas son el resultado de las actividades humanas, como son, la combustión de combustibles fósiles, los procesos industriales, la fabricación de cemento y durante la combustión de biomasa. La generación de energía y el sector industrial son unas de las fuentes de mayor volumen de emisión, esto hace que sean foco para procesos de captura de $\mathrm{CO}_{2}$.

Actualmente la mayoría de las fuentes de $\mathrm{CO}_{2}$ poseen una concentración menor al 15\%, sin embargo, hay una pequeña porción de industrias que basan su funcionamiento en los combustibles fósiles los cuales generan una concentración de hasta el $95 \%$ de $\mathrm{CO}_{2}$. Para la captación de $\mathrm{CO}_{2}$ es recomendable la implementación al sistema de fuentes con alta concentración de $\mathrm{CO}_{2}$, ya que solo requerirían de un proceso de deshidratación y compresión, así de esta misma manera evaluar los lugares de emisión y almacenamiento, ya que la distancia entre ellos puede influir en el proceso.

Algunas de las soluciones planteadas para la obtención del $\mathrm{CO}_{2}$ es el proceso de captura de este del ambiente mediante la utilización de una planta dedicada los procesos de captura de $\mathrm{CO}_{2}$, otra solución al requerimiento de este gas es la compra a empresas que se encarguen de ejecutar este tipo de procesos.

Para la captación de $\mathrm{CO}_{2}$ es fundamental evaluar las fuentes de emisión que representan altas concentraciones, los potenciales prospectos para la implementación de un proceso de captación de $\mathrm{CO}_{2}$, serían las centrales hidroeléctricas y otros procesos industriales a gran escala, cementera, plantas termoeléctricas o la captación del $\mathrm{CO}_{2}$ liberado en los procesos de refinación de hidrocarburos en refinerías. La finalidad del proceso es obtener $\mathrm{CO}_{2}$ casi puro para su transporte y posterior almacenamiento.

\section{Conclusiones}

La aplicación de la tecnología seria propuesta para suplir un déficit de gas en el país, en la actualidad Colombia, presenta un equilibrio entre la oferta y la demanda eléctrica. Colombia requeriría de diferentes fuentes de energía las cuales mediante sus remanentes energéticos pudiesen garantizar la operación continua de la planta para la generación de gas metano.

En Colombia, se observan las limitantes en cuanto a la obtención de energías a partir de fuentes renovables; por esta razón, las hidroeléctricas dominan el sector energético como el centro de atención para la aplicación de la tecnología Power to Gas.
La implementación de cualquier tecnología enfocada en el uso de fuentes de energía renovable, en todo aspecto, entre ellos el operacional requiere que Colombia adquiera un compromiso para el desarrollo de estas, a través de; inversión financiera y políticas favorables.

\section{Bibliografía}

1. Acevedo Castro, O. L., Jaramillo, J. E., \& Abril Blanco, H. (2013). ESTIMACIÓN DE EMISIONES DE GEI (CO 2 Y CH 4) GENERADAS DURANTE EL TRANSPORTE DE GAS NATURAL EN COLOMBIA, APLICANDO METODOLOGÍA IPCC. Revista Fuentes, El Reventón Energético, 11(2).

2. CHBC, California Hydrogen Bussiness Council. (2015). Power-to-Gas: The Case for Hydrogen. Los Angeles, CA 90049.

3. DNV KEMA Energy \& Sustainability. (2013). Systems Analyses Power To Gas.

4. Giraldo, L., Calle, A., \& Pineda, C. (2009). Discriminación de gas comercial y gas fizz mediante análisis de atributos avo y modelado de substitución de fluidos. Revista Fuentes, 7(2).

5. Gomez, H. S. (2001). EL RETO DEL PETROLEO EN COLOMBIA. Revista Fuentes, 1(1).

6. Götz, M. Bajohr, S. Kolb, T. Lefebvre, J. Reimert, R. (2014). Improvement of three-phase methanation reactor performance for steadystate and transient operation. Fuel processing technology, 132, 83-90.

7. Hasimoto, K. Kumagai, N. Izumiya, K. Takano, H. Shinomiya, H. Sasaki, Y. Yoshida, T. Kato, Z. (2016).The use of renewable energy in the form of methane via electrolytic hydrogen generation using carbon dioxide as the feedstock. Applied Surface Science, 308(B), 608-615.

8. Hernandez, C. (2011). Análisis ambiental de las grandes centrales hidroeléctricas de Colombia aplicando metodología multiobjetivo (tesis de pregrado). Universidad de La Salle, Bogotá D.C, Colombia

9. Intergovernmental Panel on Climate Change, IPCC. (2005). Special Report on carbon dioxide capture and storage. NY, United States of America: Cambridge University Press. 
10. PROMIGAS. (2017). Informe Del Sector Gas Natural Colombia 2017 cifras 2016 XVIII. Recuperado de http://www.promigas.com/

11. Rönsch, S. Schneider, J. Matthischke, S. Schlüter, M. Götz, M. Lefebvre, J. Prabhakaran, P. Bajohr, S. (2015). Review on methanation from fundamentals to current projects. Fuel, $166,276-296$.

12. Schaaf, T. (2014). Methanation of CO2 - storage of renewable energy in a gas distribution system. Energy, Sustainability and Society. doi: 10.1186/s13705-014-0029-1.

13. Sterner, M. (2009). Bioenergy and renewable power methane in integrated 100\% renewable energy systems (tesis de doctorado). Universidad de Kassel, Kassel, Alemania.

14. UPME, Subdirección de Energía Eléctrica - Grupo de Generación. (2016). Informe mensual de variables de generación y del mercado eléctrico colombiano. Recuperado de http:// www.upme.gov.co/

15. UPME, Subdirección de Energía Eléctrica - Grupo de Generación. (2016). Balance gas Natural 20162025. Recuperado de http://www.upme.gov.co/

16. Vaca, H. Y.(2001). PARADOJADE LAECOPETROL HOY. Revista Fuentes, 1(1).

Recepción: 1 de Febrero de 2018

Aceptación: 30 de Mayo de 2018 